\title{
Disseminated craniospinal low-grade glioma in a patient with NF-1 without optic pathway pathology: illustrative case
}

\author{
Alan R. Tang, BA, ${ }^{1}$ Joseline Haizel-Cobbina, MBChB, MPH, ${ }^{2}$ Paisit Paueksakon, MD, MPH, ${ }^{3}$ Asha Sarma, MD, ${ }^{4}$ Julie Bennett, MD, ${ }^{5}$ \\ Adam J. Esbenshade, MD, MSCl, ${ }^{6}$ and Michael C. Dewan, MD, $\mathrm{MSCl}^{2,7}$ \\ ${ }^{1}$ Vanderbilt University School of Medicine, Nashville, Tennessee; ${ }^{2}$ Vanderbilt Institute of Global Health, Vanderbilt University Medical Center, Nashville, Tennessee; Departments \\ of ${ }^{3}$ Pathology, ${ }^{4}$ Radiology, ${ }^{6}$ Pediatric Hematology-Oncology, and ${ }^{7}$ Neurological Surgery, Vanderbilt University Medical Center, Nashville, Tennessee; and ${ }^{5}$ Department of \\ Hematology/Oncology, The Hospital for Sick Children, Toronto, Ontario, Canada
}

\begin{abstract}
BACKGROUND Neurofibromatosis type 1 (NF-1) is a neurocutaneous autosomal dominant disorder that predisposes patients to develop intracranial low-grade gliomas (LGGs). Most LGGs in patients with NF-1 involve the optic pathway but can arise anywhere throughout the central nervous system. NF-1-related disseminated pediatric LGG (dPLGG) in the absence of a dominant optic pathway glioma has not been described.

OBSERVATIONS The authors discussed a case of a 10-year-old boy who presented with consideration for biopsy with nonoptic pathway PLGG with craniospinal dPLGG in the setting of NF-1. The patient's primary lesion, located in the right medulla, was initially treated with surveillance before induction chemotherapy with carboplatin and vincristine was initiated. However, surveillance imaging demonstrated significant increase in size and enhancement, and subsequent craniospinal imaging demonstrated extensive nodular dissemination in the cervicothoracic spine. A biopsy and molecular testing were subsequently performed to further evaluate the tumor, and the patient was diagnosed with dPLGG with CDKN2A deletion.

LESSONS Thorough craniospinal magnetic resonance imaging evaluation and biopsy in nonoptic pathway-dominant brain lesions in NF-1 are warranted in patients with atypical clinical and radiological findings in whom standard chemotherapeutic therapy fails.
\end{abstract}

https://thejns.org/doi/abs/10.3171/CASE21378

KEYWORDS neurofibromatosis type 1; non-optic glioma; disseminated pediatric low-grade glioma

Neurofibromatosis type 1 (NF-1) is a neurocutaneous autosomal dominant disorder affecting 1 in 2,500 to 3,000 newborns, with a wide spectrum of presentations and disease severities. ${ }^{1}$ Common manifestations include café-au-lait macules, neurofibromas (benign tumors of the peripheral nerve sheath), iris hamartomas (Lisch nodules), axillary and inguinal freckling, and skeletal deformities. ${ }^{2}$ Children with NF-1 are prone to developing pediatric low-grade gliomas (PLGG), most of which involve the optic pathway. ${ }^{3}$

PLGG is the most common form of pediatric brain tumor, comprising nearly $40 \%$ of all cases. ${ }^{4}$ Leptomeningeal disseminated PLGG (dPLGG) occurs in $3 \%-10 \%$ of cases. ${ }^{5}$ Although most often sporadic, patients with NF-1 have been noted to have increased risk for developing dPLGG. ${ }^{6,7}$ Previously, dPLGG in NF-1 has only been reported in the setting of a dominant optic pathway glioma. In NF-1, dominant nonoptic pathway gliomas are uncommon, and widespread craniospinal dissemination has yet to be reported. ${ }^{8}$ We present a case of nonoptic pathway PLGG with craniospinal dPLGG in a child with NF-1.

\section{Illustrative Case}

\section{History and Examination}

A 10-year-old boy adopted at age 4 received neurosurgical evaluation in the winter of 2021 for consideration of biopsy of a progressively enlarging and enhancing intrinsic lesion in the right medulla. He was diagnosed by clinical features with NF-1 years earlier, with physical examination findings that included a prominent left periorbital plexiform

ABBREVIATIONS dPLGG = disseminated pediatric LGG; LGG = low-grade glioma; MRI = magnetic resonance imaging; NF-1 = neurofibromatosis type 1; $P L G G$ = pediatric LGG

INCLUDE WHEN CITING Published November 1, 2021; DOI: 10.3171/CASE21378.

SUBMITTED July 14, 2021. ACCEPTED September 22, 2021.

(C) 2021 The authors, CC BY-NC-ND 4.0 (http://creativecommons.org/licenses/by-nc-nd/4.0/). 
neurofibroma, multiple café-au-lait spots, and axillary and inguinal freckling. Surveillance magnetic resonance imaging (MRI) in the winter of 2019 demonstrated a 4-mm enhancing lesion in the right medulla but no evidence of optic pathway pathology. Across serial scans, the medullary lesion grew over the subsequent 9 months. Despite the patient presenting asymptomatically with the lesion, an LGG was suspected. Given its growth and increased enhancement (and the morbidity associated with surgery), medical management was pursued. Induction chemotherapy was initiated in the fall of 2020 with carboplatin and vincristine. However, after completion of the patient's chemotherapy course, surveillance MRI in the winter of 2021 demonstrated significant increase in size and enhancement of the medullary lesion (Fig. 1). Despite standard chemotherapy, the paradoxical radiographic progression raised suspicion for a more aggressive tumor biology than originally supposed. Whole craniospinal imaging revealed likely disseminated disease: extensive nodular signal abnormality and enhancement along the cauda equina nerve roots and scattered linear leptomeningeal enhancement in the cervicothoracic spine. Innumerable nerve sheath tumors were also considered in the differential but were believed to be much less likely because they are extremely rare in cases of NF-1 compared with NF-2 and schwannomatosis. Regardless, the unusual and aggressive behavior of the CNS lesions warranted a pathologic diagnosis to guide optimal second-line therapy and tumor control. Thus, after extensive multidisciplinary discussion and careful consideration of the risks, an open brainstem biopsy was recommended.

\section{Operation and Pathological Examination}

A right-sided far lateral craniotomy and partial C1 lateral mass removal for open excisional biopsy of right medullary tumor with neuromonitoring was performed. After dural opening and gentle transposition of the vertebral artery, a peculiar sheet with a "metallic shavings" appearance was observed along the brainstem leptomeninges (Fig. 2). A segment of this leptomeninges was carefully stripped from the surface of the medulla and sent for pathologic analysis. Neuronavigation was used to identify the site of maximal enhancement, which correlated well with the microscopic piaarachnoid findings. Next, a linear incision in the craniocaudal plane was made in the dorsolateral medulla, and microinstruments were used to carefully carve a section of abnormal tissue from the center of the lesion. During the procedure, no changes to intraoperative motor evoked or somatosensory evoked potentials or to brainstem auditory evoked potentials were observed. The biopsy was uncomplicated, and the patient was discharged home at his neurological baseline on the third postoperative day. Pathologic analysis of the parenchymal sample demonstrated a low-grade pilocytic neoplasm with CDKN2A homozygous deletion. Additional molecular studies demonstrated wild-type status for BRAF p.V600E, IDH1 p.R132H, $A T R X$, and $\mathrm{H} 3$ p.K27M, and absent BRAF rearrangement or tandem duplication. The leptomeningeal sample illustrated melaninladen macrophages and neoplastic astrocytes. The patient was diagnosed with $\mathrm{dPLGG}$ with CDKN2A deletion, and treatment with temozolomide, irinotecan, and bevacizumab was initiated. At his
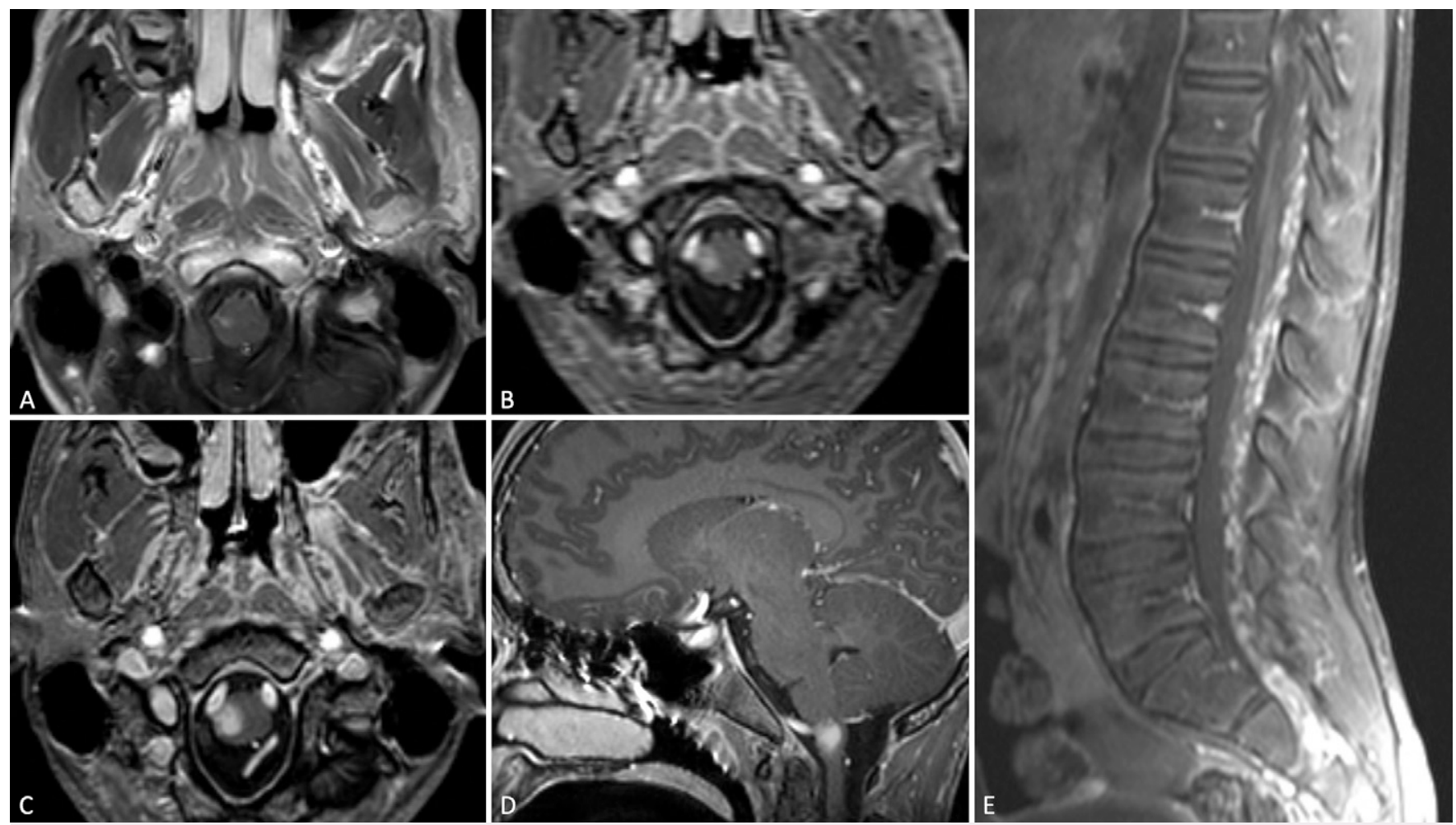

FIG. 1. Serial MRI of NF-1-associated dPLGG with CDKN2A mutation. All sequences are T1-weighted postgadolinium scans. A: Initial December 2019 axial image showing a circumscribed 4-mm lesion in the caudal medulla. B: Interval August 2020 axial image immediately before initiation of chemotherapy showing growth of the lesion $(7 \mathrm{~mm})$. Postchemotherapy February 2021 axial (C) and sagittal (D) images demonstrating continued growth of the lesion $(10 \mathrm{~mm})$ despite chemotherapy. E: Postchemotherapy February 2021 sagittal image of the lumbar spine demonstrating nodular enhancement along cauda equina nerve roots, concerning for spinal leptomeningeal dissemination. 

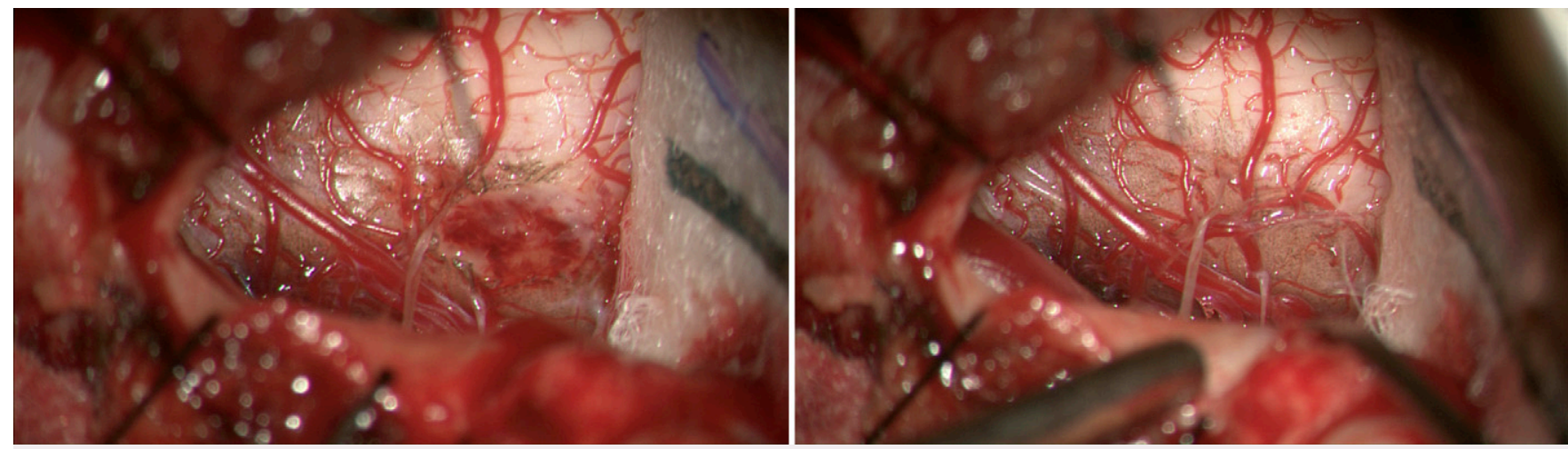

FIG. 2. Intraoperative brainstem leptomeningeal abnormalities resembling "metallic shavings." Pathology ultimately confirmed leptomeningeal dissemination of tumor.

6-month follow-up appointment, the patient was clinically and radiographically stable.

\section{Discussion \\ Observations}

To our knowledge, we present the first report of dPLGG in a patient with NF-1 without a primary optic pathway glioma. Additionally, enhancing spinal leptomeningeal nodules and tumor progression on first-line therapy represent uncommon findings in NF-1-related PLGG. Despite the elevated lifetime risk for tumors in individuals with NF-1, current guidelines do not recommend surveillance brain MRI or pathological confirmation of typical radiological findings as part of the routine care of patients of any age who have asymptomatic NF-1. Nonoptic pathway PLGG in patients with NF-1 is often asymptomatic and, therefore, may go undetected. ${ }^{8,9}$ When $\mathrm{MRI}$ is performed, it is occasionally difficult to distinguish atypical NF-1-related myelin vacuolization or spongiform changes from PLGG, especially at a single time point, because of overlap in imaging characteristics. ${ }^{9-11}$

Nonoptic pathway PLGG in NF-1 is an understudied subset. Up to $15 \%$ of all patients with NF-1 present with cerebral tumors in the first two decades of life, most of which are low-grade pilocytic astrocytomas. ${ }^{10,12} \mathrm{NF}-1$ has been shown to act as a positive prognostic factor for progression-free survival in LGG. ${ }^{13}$ Specifically, NF-1-associated brainstem gliomas have a lower frequency of progression, respond more favorably to chemotherapy treatment, and demonstrate a more favorable prognosis and event-free survival rate than their non-NF1-associated counterparts. ${ }^{13-16}$ Treatment for NF-1 LGG is multifaceted and hinges on both lesion location and symptomatology. Tumors involving the optic pathway are often treated when visual function is impaired, either through resection or chemotherapy with a regimen of carboplatin and vincristine or vinblastine, which is considered standard of care. Less commonly, resection or debulking is warranted; radiation is avoided because of risk of secondary tumor development. $^{13,17,18}$ However, controversy remains regarding treatment versus observation in patients with asymptomatic pediatric NF-1 LGG. Because LGG in patients with NF-1 has been associated with spontaneous regression, serial observation is often preferred, and a complete metastatic examination is rarely performed. ${ }^{19,20}$

As we learn more about the underpinnings of the molecular drivers in LGG, the need for proper genetic characterization of these tumors is becoming clear. LGGs in cases of NF-1 have historically been thought to harbor a single genetic alteration. Recent studies have shed new light and suggested that additional alterations in driver genes can be seen, including co-occurrence of BRAF p.V600E, FGFR1 p.N546K, and H3 p.K27M mutations. ${ }^{21,22}$ It is well known that tumors with $\mathrm{H} 3$ p.K27M mutation are aggressive; however, further study is needed to understand the clinical behavior of tumors with additional alterations within the RAS/MAPK pathway. Low-grade lesions may also show copy number gains of TERT, in contrast to highgrade lesions, which may show loss of $C D K N 2 A / B$ or inactivation mutations of ATRX and TP53. ${ }^{23}$ Taken together, these data suggest that molecular analysis of tumors in cases of NF-1 (particularly those that are located outside of the optic pathway) is crucial because it may allow early detection of more aggressive tumors.

This illustrative case highlights the value of thorough craniospinal MRI evaluation and biopsy in nonoptic pathway-dominant brain lesions in patients with NF-1. Specifically, patients with progressive enlargement and enhancement in whom standard chemotherapeutic therapy fails should be considered at high clinical risk for unpredictable natural history.

\section{Lessons}

Although patients with NF-1 generally have more predictive molecular pathology than their nonsyndromic counterparts, nonoptic pathway-dominant suspected NF-1-associated PLGGs that progress on standard therapy should raise suspicion for unique disease patterns. There remains an important role for craniospinal imaging in patients with nonoptic pathway lesions, noting that dissemination of low-grade PLGG is possible, even in the setting of NF-1. Biopsy should be considered in cases with atypical clinical and radiological findings because specific molecular features such as $C D K N 2 A$ deletion can be instrumental in guiding second-line therapy and tighter radiographic follow-up.

The take-home lesson from this case is twofold. First, patients with NF-1-associated PLGG are at risk for dissemination, just like their non-NF-1 counterparts. Second, atypical radiographic findings should raise the index of suspicion for atypical tumor biology. The medullary lesion in our patient grew despite standard chemotherapy and was noted in the setting of nonoptic pathway-associated dissemination. The subsequent identification of a high-risk mutation 
(CDKN2A deletion) should reframe the nature in which second-line therapy is administered and warrant closer monitoring.

\section{References}

1. Hirbe AC, Gutmann DH. Neurofibromatosis type 1: a multidisciplinary approach to care. Lancet Neurol. 2014;13(8):834-843.

2. Cimino PJ, Gutmann DH. Neurofibromatosis type 1. Handb Clin Neurol. 2018;148:799-811.

3. Campen CJ, Gutmann DH. Optic pathway gliomas in neurofibromatosis type 1. J Child Neurol. 2018;33(1):73-81.

4. Chalil A, Ramaswamy V. Low grade gliomas in children. J Child Neurol. 2016;31(4):517-522.

5. Tabori U, Rienstein S, Dromi Y, et al. Epidermal growth factor receptor gene amplification and expression in disseminated pediatric low-grade gliomas. J Neurosurg. 2005;103(4 suppl):357-361.

6. Perilongo G, Garrè ML, Giangaspero F. Low-grade gliomas and leptomeningeal dissemination: a poorly understood phenomenon. Childs Nerv Syst. 2003;19(4):197-203.

7. Landry JP, Schertz KL, Chiang YJ, et al. Comparison of cancer prevalence in patients with neurofibromatosis type 1 at an academic cancer center vs in the general population from 1985 to 2020. JAMA Netw Open. 2021;4(3):e210945.

8. Sellmer L, Farschtschi S, Marangoni M, et al. Non-optic glioma in adults and children with neurofibromatosis 1. Orphanet $J$ Rare Dis. 2017;12(1):34.

9. Ferner RE, Huson SM, Thomas N, et al. Guidelines for the diagnosis and management of individuals with neurofibromatosis 1. J Med Genet. 2007;44(2):81-88.

10. Fisher MJ, Jones DTW, Li Y, et al. Integrated molecular and clinical analysis of low-grade gliomas in children with neurofibromatosis type 1 (NF1). Acta Neuropathol. 2021;141(4):605-617.

11. Packer RJ, lavarone A, Jones DTW, et al. Implications of new understandings of gliomas in children and adults with NF1: report of a consensus conference. Neuro Oncol. 2020;22(6):773-784.

12. Hernáiz Driever $P$, von Hornstein $S$, Pietsch $T$, et al. Natural history and management of low-grade glioma in NF-1 children. J Neurooncol. 2010;100(2):199-207.

13. Helfferich J, Nijmeijer R, Brouwer OF, et al. Neurofibromatosis type 1 associated low grade gliomas: a comparison with sporadic low grade gliomas. Crit Rev Oncol Hematol. 2016;104:30-41.

14. Pollack IF, Shultz B, Mulvihill JJ. The management of brainstem gliomas in patients with neurofibromatosis 1. Neurology. 1996;46(6): 1652-1660.

15. Ater JL, Xia C, Mazewski CM, et al. Nonrandomized comparison of neurofibromatosis type 1 and non-neurofibromatosis type 1 children who received carboplatin and vincristine for progressive low-grade glioma: a report from the Children's Oncology Group. Cancer. 2016;122(12):1928-1936.
16. Ater JL, Zhou T, Holmes E, et al. Randomized study of two chemotherapy regimens for treatment of low-grade glioma in young children: a report from the Children's Oncology Group. J Clin Oncol. 2012;30(21):2641-2647.

17. Yamanaka R, Hayano A. Radiation-induced malignant peripheral nerve sheath tumors: a systematic review. World Neurosurg. 2017; 105:961-970.e8.

18. Packer RJ, Ater J, Allen J, et al. Carboplatin and vincristine chemotherapy for children with newly diagnosed progressive low-grade gliomas. J Neurosurg. 1997;86(5):747-754.

19. Rozen WM, Joseph S, Lo PA. Spontaneous regression of lowgrade gliomas in pediatric patients without neurofibromatosis. Pediatr Neurosurg. 2008;44(4):324-328.

20. Perilongo G, Moras $P$, Carollo $C$, et al. Spontaneous partial regression of low-grade glioma in children with neurofibromatosis-1: a real possibility. J Child Neurol. 1999;14(6):352-356.

21. Ryall S, Zapotocky M, Fukuoka K, et al. Integrated molecular and clinical analysis of 1,000 pediatric low-grade gliomas. Cancer Cell. 2020;37(4):569-583.e5.

22. Mahdi J, Goyal MS, Griffith J, Morris SM, Gutmann DH. Nonoptic pathway tumors in children with neurofibromatosis type 1. Neurology. 2020;95(8):e1052-e1059.

23. D'Angelo F, Ceccarelli M, Tala, et al. The molecular landscape of glioma in patients with neurofibromatosis 1. Nat Med. 2019;25(1): 176-187.

\section{Disclosures}

The authors report no conflict of interest concerning the materials or methods used in this study or the findings specified in this paper.

\section{Author Contributions}

Conception and design: Tang, Haizel-Cobbina, Paueksakon, Esbenshade, Dewan. Acquisition of data: Tang, Sarma, Esbenshade. Analysis and interpretation of data: Tang, Paueksakon, Sarma, Bennett, Esbenshade, Dewan. Drafting the article: Tang, Haizel-Cobbina, Paueksakon, Sarma, Esbenshade. Critically revising the article: Tang, Haizel-Cobbina, Sarma, Bennett, Esbenshade, Dewan. Reviewed submitted version of manuscript: Tang, Haizel-Cobbina, Sarma, Bennett, Esbenshade, Dewan. Approved the final version of the manuscript on behalf of all authors: Tang. Administrative/technical/ material support: Haizel-Cobbina, Paueksakon. Study supervision: Dewan.

\section{Correspondence}

Alan R. Tang: Vanderbilt University School of Medicine, Nashville, TN. alan.r.tang@vanderbilt.edu. 